Results The sensitivity of this magnetic LFIA of NT-proBNP was $0.01 \mathrm{ng} / \mathrm{ml}$, the detection range was reached five orders of magnitude, and the detection time was within $15 \mathrm{~min}$.

Conclusion It was showed that this magnetic LFIA of NT-proBNP has a high sensitivity, wide detection range and short detection time. It is a simple, rapid, accurate, quantitative and point-of-care testing which deserve to be spread and industrialised.

\section{e0680 RELATIONSHIP BETWEEN POLYMORPHISM OF ACE AND THE CURATIVE EFFECT OF METOPROLOL ON CHRONIC HEART FAILURE}

doi:10.1136/hrt.2010.208967.680

Miao Peizhi, Gu Shuiming. Shanghai Xuhui Central Hospital, Department of Cardiology, Shanghai, China

Objective To investigate the effect of metoprolol on chronic heart failure and to discuss the relationship between polymorphism of ACE and the curative effect of metoprolol.

Methods 118 patients with chronic heart failure were included randomly and were divided into two groups. In control group (group A), the patients were treated with benazapril 2.5-10 mg once daily and routinely, in treatment group the patients were treated additionally with metoprolol $12.5-100 \mathrm{mg}$ once daily. The period of treatment for all patients was 2 years. ACE polymorphism was detected by a PCR.

Results The patients encountered heart failure, myocardial infarction, malignant arrhythmia and sudden death in metoprolol group were lower than those of control group, the difference was significant $(p<0.05)$. PRA was significant increased and AngII, ALD were significant lowered in two groups after treatment. The frequency of DD genotype in metoprolol effective group was 0.47 and that in metoprolol ineffective group was 0.13 , the difference was significant $(p<0.05)$. The effective rate of DD genotype was $90.9 \%$, that of ID genotype was $76.9 \%$, that of II genotype was $54.2 \%$, and the effective rate of DD genotype was significantly higher than II genotype $(\mathrm{p}<0.05)$.

Conclusion Adding metoprolol to the treatment of chronic heart failure can improve heart function. The polymorphism of the ACE (I/D) is helpful for the diagnosis of the therapeutic efficacy of metoprolol in chronic heart failure.

\section{e0681 EFFECT OF CHEMOTATIC FACTOR FKN ON NF-KB AND TNF- $\alpha$ EXPRESSION IN PERIPHERAL BLOOD MONOCYTES AND THE ROLE OF PI3K}

doi:10.1136/hrt.2010.208967.681

Sun Jian, Guo Hui-jiao, Lei Ming-ming, Hou Wen-li, Yang Chun-yan, Wu Zhe. The First Hospital of Ji lin University, Chang chun, China

Objective In the present study, the effect of Fractalkine (FKN) on the expression of NF- $\mathrm{KB}$ and TNF- $\alpha$ induced by FKN was investigated one of possible signal transduction pathways of FKN/CX3CR1 in atherosclerosis, and the role of PI3K were also investigated.

Methods 1) Peripheral blood monocytes were isolated from fresh blood of healthy volunteers by Ficoll-Paque gradient centrifugation. 2) Divide the extractive peripheral blood monocytes into four groups :control group, FKN group, LY294002 group and PDTC group. 3) Measure the NF- $\mathrm{BB}$ expression of monocytes from each group by Western Blot. 4) Collect the supernatant of monocytes from each group, determine the expression of TNF- $\alpha$ by ELISA.

Result 1. The expression of NF-KBand TNF- $\alpha$ in FKN group was increased, compared with that control group $(p<0.05)$. 2 . The expression of NF- $\kappa$ B and TNF- $\alpha$ in LY294002 group was decreased, compared with that FKN group $(\mathrm{p}<0.05)$.
Conclusions FKN-CX3CR1 increase the expressions of NF- $\mathrm{KB}$ and TNF- $\alpha$ in peripheral blood monocytes, which may be one of the mechanisms of contributing to the progression of atherosclerosis; After interacting with its receptor CX3CR1, FKN activates the PI3K by coupled with G-protein and then initiates intracellular signal conductive mechanism.

\section{Q0682 THE COMBINED USE OF UROKINASE AND GLYCOPROTEIN IIB/IIIA-TARGETED MICROBUBBLES RECANALIZE RABBIT FEMORAL ARTERY WITH THROMBOTIC OCCLUSIONS}

doi:10.1136/hrt.2010.208967.682

Yu-ming Mu, Li-Na Guan, Ling Li, Chao-Feng Guo, Chun-Me Wang. Department of Echocardiography, Center of the Medical Ultrasound, The First Affiliated Hospital of Xinjiang Medical University

Objective To determine the effect of the combined use of urokinase and glycoprotein IIb/IIIa-targeted microbubbles prepared by direct conjugation method to dissolve the thromb.

Methods Urokinase and RGDS were in conjunction with microbubbles (SonoVue) by the direct conjugation method. The size, shape, fluorescent intensity, the binding rates of Urokinase and RGDS, and the activity of Urokinase were measured and analysed. A total of 42 rabbits with platelet-rich thrombi in the femoral artery were randomised into seven treatment groups ( $\mathrm{n}=6)$ : 1) ultrasound alone (US); 2) ultrasound plus non-targeted microbubbles (US+M); 3) urokinase alone (UK); 4) ultrasound, non-targeted microbubble and urokinase (US+M+UK); 5) ultrasound plus RGDS microbubble (US+R); 6) RGDS microbubble plus urokinase (R+UK); and 7) ultrasound, RGDS microbubble and urokinase (US+R+UK). US in diagnostic ultrasound were simultaneously applied over the thrombus up to $30 \mathrm{~min}$. The thrombolytic effect was evaluated at 120 min post treatment

Results SonoVue, Urokinase and RGDS were combined successfully. In vitro thrombolysis experiment indicated that the urokinase in the prepared contrast agent had activity $(p<0.01)$. For US, UK, US $+M$, $\mathrm{US}+\mathrm{R}$ and US $+\mathrm{M}+\mathrm{UK}$ groups, recanalisation was failed with the blood flow less than $15 \%$ of baseline. The blood flow for $\mathrm{R}+\mathrm{UK}$ was $15 \% \sim 49 \%$ of baseline, while that of US $+\mathrm{R}+\mathrm{UK}$ was more than $75 \%$ of baseline $(\mathrm{p}<0.001)$

Conclusion The combined use of urokinase and glycoprotein IIb/ IIIa-targeted microbubbles is effective in targeting thromb and recanalizing thrombolytic occlusion.

\section{e0683 CLINICAL SIGNIFICANCE OF AUTOANTIBODIES AGAINST CARDIAC TROPONIN I IN PATIENTS WITH MYOCARDIAL INFARCTION AND CHRONIC HEART FAILURE}

doi:10.1136/hrt.2010.208967.683

Yang Di, Bian Zhiping, Ji Peng, Li Bing, Chen Xiangjian, Xu Jindan, Gu Chunrong, Zhang Jinan. Institute of Cardiovascular Disease, First Affiliated Hospital of Nanjing Medical University, Nanjing

Objective Autoantibodies against cardiac troponin I (cTnI) have been described in the serum from patients with dialated cardiomyopathy and heart failure. The clinical significance of these autoantibodies remains unknown. The present study was designed to evaluate the relationship between the serum level of autoantibodies against cardiac troponin I and the prognosis of patients with myocardial infarction (MI) and chronic heart failure (CHF).

Methods 97 patients were studied in the present study, including 38 patients (68.3 \pm 7.9 years, 28 males) with $\mathrm{MI}$ and 59 patients (63.3 \pm 14.6 years, 44 males) with CHF. The patients were recruited in the First Affiliated Hospital of Nanjing Medical University from 2005 to 2008.78 healthy control subjects were enrolled in the study. The 